\title{
Mathematical modeling of a temperature-sensitive and tissue-mimicking gel matrix
}

\author{
Baeckkyoung SUNG ${ }^{1}$ \\ ${ }^{1}$ KIST Europe Forschungsgesellschaft mbH
}

May 5, 2020

\begin{abstract}
Programmed to retain active responsivity to environmental stimuli, diverse types of synthetic gels have been attracting interests regarding various applications, such as versatile elastic biodevices. In a different approach, when the gels are made of tissuederived biopolymers, they can act as an artificial extracellular matrix (ECM) for use as soft implants in regenerative medicine. To explore the physical properties of hydrogels in terms of statistical thermodynamics, the mean-field Flory-Huggins-Rehner theory has long been used with various analytical and numerical modifications. Here we suggest a novel mathematical model on the volume phase transition of a biological hybrid gel that is sensitive to ambient temperature. To mimic acellular soft tissues, the ECM-like hydrogel is modeled as a network of biopolymer chains, such as type I collagen and gelatin, which are covalently crosslinked and swollen in aqueous solvents. Within the network, thermoresponsive synthetic polymer chains are doped by chemical conjugation. Based on the Flory-Huggins-Rehner framework, our model phenomenologically illustrates a wellcharacterized volume phase behavior of engineered tissue mimics as a function of temperature by formulating the ternary mixing free energy of the polymer-solvent system and by generalizing the elastic free energy term. With this formalism, the decoupling of the Flory-Huggins interaction parameter between the thermoresponsive polymer and ECM biopolymer enables deriving a simple analytical equation for the volume phase transition as a function of the structural and compositional parameters. We show that the doping ratio of the thermoresponsive polymers affects the phase transition temperature of the ECM-like gels.
\end{abstract}

\section{Introduction}

Polymer gel is defined as an elastic random network of flexible chains in a liquid solution ${ }^{1}$. In the fields of pharmacy and medicine, three-dimensional (3D) polymeric gel matrices which are swollen in aqueous solvents, termed as hydrogels, have been widely used for drug delivery and tissue scaffolding ${ }^{2}$. Tissue mimicry is achieved when tissue-derived biopolymers, including collagen and gelatin, are adopted as the base material for the gel fabrication, which serves as an engineered extracellular matrix (ECM), providing tunable physicochemical properties with complete cytocompatibility ${ }^{3}$. In a different viewpoint, when a hydrogel is synthesized with thermoresponsive polymers, such as poly(N-isopropylacrylamide) (pNIPAM) and its copolymers, the reversible swelling-deswelling behavior can be conferred to the hydrogel matrix in a temperature-sensitive manner ${ }^{4}$.

Thermodynamic understanding of the polymeric gel system has been achieved using a mean-field approach, called the Flory-Huggins-Rehner theory ${ }^{5-8}$. In this lattice theory, if we limit the scope for non-ionic gels, the total Gibbs free energy of the system is a summation of two main contributions: the free energy of mixing between polymer segments and solvent molecules, and the elastic free energy of crosslinked polymer network. In the former, the polymer-solvent interaction is represented by a non-dimensional multiplication coefficient, called the Flory-Huggins interaction parameter. The Flory-Huggins parameter reflects both entropic and enthalpic interactions, and the temperature responsivity of a polymer chain or network is mediated by this parameter. Thermoresponsive hydrogels have primarily been modeled for pNIPAM systems, considering 
binary mixing free energy between polymer and solvent ${ }^{9}$. Because a single chain of pNIPAM exhibits coil-toglobule transition above lower critical solution temperature, the pNIPAM gel collapses in the same critical temperature. This reversible collapsing is called volume phase transition, which is a first-order-like phase transition. A variety of biosensors and drug delivery platforms have been developed based on the deswelling (shrinking) behavior of miniaturized pNIPAM gels. Nevertheless, synthetic stimuli-responsive polymers, including pNIPAM, predominantly have few tissue-relevant features ${ }^{10}$.

Hence it is strongly required to use the tissue-derived biopolymers for implementing artificial ECMs, albeit those biopolymers are lacking thermoresponsivity in the room temperature range. To overcome this limitation, doping pNIPAM chains in the biopolymer network through chemical conjugations can be an efficient way to combine the advantages of both types of polymers in a single gel matrix. In this work, we theoretically design a new type of engineered ECM-like hydrogel where thermoresponsive synthetic polymer chains are doped in a 3D ECM biopolymer gel (Fig. 1). Considering the ternary mixing free energy of the biopolymer-solvent system and by assuming a generalized form of the gel elastic free energy, we derive a simple analytical relation to show well-defined volume phase behaviors of the tissue-resembling gel as a function of temperature. We calculate optimized numerical parameter sets of the hybrid gel matrix for biomedical applications in vivo andin vitro, which are aimed to operate at human body temperature.

\section{Model Derivation}

The mean-field Flory-Huggins-Rhener theory enables predicting the phase behavior of gel matrices, providing an intuitive picture of the underlying statistical thermodynamics of a solution system ${ }^{8}$. In this theory, the thermodynamic mechanism of gel volume phase transition (swelling and deswelling) is described as an interplay between mixing free energy of the binary mixture (i.e., polymer and solvent) and elastic free energy of the solid-like polymer network ${ }^{9}$.

The free energy of mixing is hypothesized to be quantified using a liquid lattice model of $n$ unit cells ${ }^{11}$. The total Gibbs free energy $(\Delta \Phi)$ of the system is given by the summation of mixing free energy $\left(\Delta \Phi_{\mu \imath \xi}\right)$ and elastic free energy $\left(\Delta \Phi_{\epsilon \lambda}\right)$ :

$$
F=F_{\mathrm{mix}}+F_{\mathrm{el}}
$$

Typically, the mixing free energy of a binary system has been considred to derive the well-known equation as below ${ }^{9}$ :

$$
F_{\text {mix }}=n k_{B} T\left[(1-\phi) \ln (1-\phi)+\chi^{\text {Flory }} \phi(1-\phi)\right],
$$

where $\varphi$ is the total polymer volume fraction and $\chi^{\Phi \lambda \circ \rho \psi}$ is the Flory-Huggins interaction parameters between the polymer and solvent, $T$ is the temperature, and $k_{B}$ is the Boltzmann constant.

The elastic free energy of the crosslinked polymer network is given by in the simplest form as follows ${ }^{9}$ :

$$
\Delta F_{\mathrm{el}}=\frac{3 k_{B} T}{2 N_{x}}\left[\left(\frac{\phi_{0}}{\phi}\right)^{\frac{2}{3}}-1-\ln \left(\frac{\phi_{0}}{\phi}\right)\right],
$$

where $\varphi_{0}$ represents the polymer volume fraction at a reference state, and $N_{x}$ is the degree of polymerization between crosslinks.

In our model, we modify the mixing free energy term as that of a ternary mixture, which means that the system comprises two types of polymers (i.e., tissue-derived biopolymer and thermoresponsive pNIPAM) and a common solvent (i.e., water). We assume that the elastic free energy is attributed to the elasticity of the 
crosslinked biopolymer network, and generalize the expression by introducing a dimensionless parameter, $a$ $>0$. Thus, the ternary mixing free energy and the elastic free energy are given by the following:

$F_{\text {mix }}=n k_{B} T\left[\frac{\phi_{\mathrm{NIPA}}}{N_{\mathrm{NIPA}}} \ln \phi_{\mathrm{NIPA}}+\left(1-\phi_{\text {bio }}-\phi_{\mathrm{NIPA}}\right) \ln \left(1-\phi_{\text {bio }}-\phi_{\mathrm{NIPA}}\right)+\chi_{\text {bio }} \phi_{\text {bio }}\left(1-\phi_{\text {bio }}-\phi_{\text {bio }}\right)+\frac{\phi_{\text {bio }}}{N_{\text {bio }}} \ln \phi_{\text {bio }}+\chi_{\mathrm{NIPA}} 9\right.$

where $n_{\mathrm{NIPA}}=n \phi_{\mathrm{NIPA}}=n \frac{V_{\mathrm{NIPA}}}{V}, n_{\mathrm{NIPA}} v_{w}=V_{\mathrm{NIPA}}, n_{\mathrm{bio}} v_{w}=V_{\mathrm{bio}}, n v_{w}=V$,

and

$$
\Delta F_{\text {el }}=\frac{k_{B} T}{N_{x}}\left[\frac{3}{2}\left\{\left(\frac{\phi_{\text {bio_i }}}{\phi_{\text {bio }}}\right)^{\frac{2}{3}}-1\right\}-\alpha \ln \left(\frac{\phi_{\text {bio_i }}}{\phi_{\text {bio }}}\right)\right]=\frac{k_{B} T}{N_{x}}\left[\frac{3}{2}\left\{\left(\frac{V}{V_{i}}\right)^{\frac{2}{3}}-1\right\}-\alpha \ln \left(\frac{V}{V_{i}}\right)\right] .
$$

Here, we denote $n$ and $v_{w}$ as the total number of lattice sites and the volume of a lattice (unit cell), respectively. In addition, $\varphi_{\beta \imath}$ and $\varphi_{N I \Pi A}$ are volume fraction of biopolymer and pNIPAM, respectively. Similarly, $V_{\text {bio }}$ and $V_{N I P A}$ are defined as the volume occupied by biopolymer and by pNIPAM, respectively. The $\chi_{\beta 10}, \chi_{\text {NIIIA }}$, and $\chi_{\beta 10-N I I A}$ are Flory-Huggins interaction parameters of biopolymer-water, pNIPAMwater, and biopolymer-pNIPAM, respectively. The initial biopolymer volume fraction $\varphi_{\beta \imath \_\_}$at the initial gel volume $V_{i}$ and the biopolymer volume fraction $\varphi_{\beta i o}$ at a given gel volume $V$ are defined as $\phi_{b i o_{-} i}=\frac{V_{\text {bio }}}{V_{i}}$ and $\phi_{\text {bio }}=\frac{V_{\text {bio }}}{V}$, respectively, where $V_{\text {bio }}$ is the volume occupied by biopolymer chains.

We assume that the Flory-Huggins interaction parameter between biopolymer and pNIPAM can be approximated as a decoupled form as follows:

$$
\chi_{b i o-N I P A} \approx \chi_{\text {bio }}+\chi_{\mathrm{NIPA}}-2 \sqrt{\chi_{\mathrm{bio}}} \sqrt{\chi_{\mathrm{NIPA}}} .
$$

Then, the partial derivative of the mixing free energy with volume can be approximated as:

$\frac{\partial F_{\mathrm{mix}}}{\partial V} \approx \frac{k_{B} T}{v_{w}}\left[\ln \left(1-\frac{V_{\mathrm{bio}}+V_{\mathrm{NIPA}}}{V}\right)+\frac{V_{\mathrm{bio}}+V_{\mathrm{NIPA}}}{V}+\chi_{\mathrm{bio}} \frac{V_{\mathrm{bio}}^{2}}{V^{2}}+\chi_{\mathrm{NIPA}} \frac{V_{\mathrm{NIPA}}^{2}}{V^{2}}+2 \sqrt{\chi_{\mathrm{bio}} \chi_{\mathrm{NIPA}}} \frac{V_{\mathrm{bio}} V_{\mathrm{NIPA}}}{V^{2}}\right] \approx \frac{k_{B} T}{v_{w}}\left(\frac{V_{i}}{V}\right)^{2}$

Additionally, the time derivative of the elastic free energy becomes:

$$
\frac{\partial F_{\mathrm{el}}}{\partial V}=\frac{k_{B} T}{N_{x} V_{i}}\left[\left(\frac{V_{i}}{V}\right)^{\frac{1}{3}}-\alpha\left(\frac{V_{i}}{V}\right)\right] .
$$

Equilibrium swelling and deswelling are calculated by assuming the balance of elastic and mixing contributions to the osmotic pressure in the gel matrix ${ }^{9,12}$. Then, the osmotic pressure is the negative time-derivative of the total free energy and equals zero when the contributions from mixing and elastic free energies are in equilibrium:

$\Pi=-\frac{\partial F}{\partial V}=-\left(\frac{\partial F_{\mathrm{mix}}}{\partial V}+\frac{\partial F_{\mathrm{el}}}{\partial V}\right)=-\left[\frac{k_{B} T}{v_{w}}\left(\frac{V_{i}}{V}\right)^{2}\left(\sqrt{\chi_{\mathrm{bio}}} \frac{V_{\mathrm{bio}}}{V_{i}}+\sqrt{\chi_{\mathrm{NIPA}}} \frac{V_{\mathrm{NIPA}}}{V_{i}}\right)^{2}+\frac{k_{B} T}{N_{x} V_{i}}\left\{\left(\frac{V_{i}}{V}\right)^{\frac{1}{3}}-\alpha\left(\frac{V_{i}}{V}\right)\right\}\right]=0$ 
This equation gives the final formula as follows:

$$
\Lambda+\Gamma\left\{\left(\frac{V}{V_{i}}\right)^{\frac{5}{3}}-\alpha\left(\frac{V}{V_{i}}\right)\right\}=0,
$$

where the coefficients $\Lambda>0$ and $\Gamma>0$ are defined as

$$
\Lambda=\frac{1}{v_{w}}\left(\sqrt{\chi_{\text {bio }}} \frac{V_{\text {bio }}}{V_{i}}+\sqrt{\chi_{\mathrm{NIPA}}} \frac{V_{\mathrm{NIPA}}}{V_{i}}\right)^{2}
$$

and

$$
\Gamma=\frac{1}{N_{x} V_{i}}
$$

Therefore, in isobaric conditions, an analytical relation between the gel deswelling parameter $V / V_{i}$ and the variables $N_{x}$ and $\chi_{\text {NIIIA }}$ is obtained.

If we substitute $\chi_{\text {NIIIA }}(T)$ as an explicit function of only temperature in a conventional expression ${ }^{12}$,

$$
\chi_{\mathrm{NIPA}}(T)=\frac{1}{2}-A\left(1-\frac{\Theta}{T}\right)
$$

the analytical relation between $T, N_{x}$, and $V / V_{i}$ is finally derived as follows:

$$
\frac{T}{\Theta}=\left[1-\frac{1}{2 A}+\frac{1}{A}\left[\frac{V_{i}}{V_{\mathrm{NIPA}}} \sqrt{\frac{1}{N_{x}} \frac{v_{w}}{V_{i}}\left\{\alpha\left(\frac{V}{V_{i}}\right)-\left(\frac{V}{V_{i}}\right)^{\frac{5}{3}}\right\}}-\frac{\sqrt{\chi_{\mathrm{bio}}}}{r_{\mathrm{polym}}}\right]^{2}\right]^{-1}
$$

where $\Theta$ is the Flory's $\Theta$ temperature of pNIPAM, $A$ is a factor which can be experimentally determined. In addition, the doping ratio of thermoresponsive polymer to tissue-derived biopolymer is defined as

$$
r_{\text {polym }}=\frac{V_{\text {NIPA }}}{V_{\text {bio }}} .
$$

Here, $0<r_{\text {polym }}$ [?] 1 , and we assume that $V_{i} / V_{N I P A}=20$ for all cases. The parameter values and plotting variables are summarized in Tables 1 and 2, which are used for the numerical graphs in Figs. 2 to 5.

\section{Results and Discussion}

Stable and well-characterized series of curves are generated from our equation, demonstrating the deswelling of the biopolymer (i.e., type I collagen) gel matrix with a temperature increase. Under a fixed crosslinking condition, the gel elasticity-associated coefficient $a$ appears to be a major factor in determining the deswelling behavior (Fig. 2). The gel shrinkage becomes more sensitive to the normalized temperature change at $T / \Theta$ $<0.95$, as decreasing $a$ value on the order of magnitude range of $a[?] 10^{9}$. When $a$ exceeds $\sim 10^{9}$, the volume change curves are no longer an injective function in the given range of normalized temperature. For the subsequent analyses, we assume $a=1.0 \times 10^{7}$ as an optimal elastic condition.

Next, we find that the doping ratio of thermoresponsive pNIPAM chains $\left(A=-6.4\right.$ and $\left.\Theta=315 \mathrm{~K}^{13}\right)$ in the type I collagen gel matrix is another critical parameter to tune the overall temperature range of the volume phase transition. Fig. 3 demonstrates that the phase transition temperature is tunable in a wide range $(\sim 50$ 
degrees) from room temperature $\left(T_{\text {room }} \sim 22{ }^{\circ} \mathrm{C}\right)$, by varying the mixing ratio of pNIPAM $\left(r_{\text {polym }}\right)$ from 0.3 to 0.5. [Note that the curves for only four values of $r_{\text {polym }}$ has been plotted in different colors in the Fig. 3 . The transition temperature tuning can be achieved in a fully continuous manner in this range.]

In a fixed doping ratio $\left(r_{\text {polym }}\right)$ of the thermosensitive component pNIPAM, it is found that the crosslinking degree of the network (represented by the degree of polymerization between crosslinks, $N_{x}$ ) is the parameter which controls the temperature sensitivity of the gel matrix. For each transition curve at different $r_{\text {polym }}$ value in Fig. 3, a higher gel volume change occurs for less crosslinked networks (i.e., larger $N_{x}$; displayed as a series of curves with decreasing saturation), when compared in a given temperature domain. The condition of $r_{\text {polym }}=0.35$ and $N_{x}=1$ has selected as an optimized physicochemical parameter set under the consideration of (1) the onset of the phase transition at a temperature slightly higher than the body temperature $\left(T_{\text {body }}\right.$ $\sim 37^{\circ} \mathrm{C}$ ) and $(2)$ the transition temperature range spanning a few degrees above $T_{\text {body }}$, which corresponds to unharmful hyperthermia for human tissues in short time scales ${ }^{14}$.

Under the selected parameter values, the effect of collagen molecular structure on the transition temperature range of the gel matrix has been investigated (Fig. 4). Because the information on the conformation of type I collagen is reflected in the Flory-Huggins interaction parameter between biopolymer and water, $\chi_{\beta}$, we plot for the four different values of $\chi_{\beta 10}$ that are chemically plausible in acellular in vitro situations. In Fig. 4 , the curve for gel matrix made of intact collagen type $\mathrm{I}\left(\chi_{\beta 10}=0.161^{15}\right)$, which is a composite of a main helical rod and extrahelical peptides at both terminals, is presented with the parameter set chosen in Fig. 3 (blue solid line). If the gel comprises the pronase-digested collagen type I ( $\left.\chi_{\beta 10}=0.107^{15}\right)$, which has no extrahelical structures to be closer to a rod particle, the phase transition range decreases about by 8 degrees (blue dashed line). When the input value of $\chi_{\beta 10}$ is for gelatin $\left(\chi_{\beta 10}=0.48\right.$ for coil state ${ }^{16}$ and $\chi_{\beta 1 o}=0.49$ for coexistence of coil and helical states ${ }^{16,17}$ ), the resulting plots (cyan solid and dashed lines, respectively in Fig. 4) illustrate that the transition temperature ranges appear to be above the temperature threshold for denaturation from type I collagen to gelatin $\left({ }^{\circ} 65^{\circ} \mathrm{C}^{18}\right)$, which partially validates the effectiveness of our model in a biophysical basis.

For bioengineering applications, the representative examples of the optimized matrix design are suggested for type I collagen and gelatin gels with different doping ratios of pNIPAM (Fig. 5). Assuming the volume ratio of the total polymers in a swollen hydrogel is about 0.1 (with $V_{i} / V_{N I P A}=20$ ), then $r_{\text {polym }}=0.35$ for collagen type I and $r_{\text {polym }}=0.48$ for gelatin are found to be suitable for inducing gel deswelling through local heating in the viable temperature range for tissues in vivo and in vitro. In these conditions, the gel matrices are fully swelled at $T_{b o d y}$ and completes deswelling at slightly augmented temperatures (i.e., $\sim 8$ degrees above $T_{b o d y}$ ) and vice versa. For instance, this property can be utilized for controlled release of drugs in human body or as miniaturized tissue-resembling actuators in organ-on-a-chip platforms for cell cultures ${ }^{10}$.

\section{Conclusion}

An analytical model is developed to quantitatively design artificial ECM gels that exhibit thermosensitive and reversible shrinking above body temperature. The modeling is based on the mean-field thermodynamic theory by Flory-Huggins-Rehner, from which an explicit equation is derived to include tunable parameters on the gel structure and elasticity, chemical composition of the gel matrix, and molecular conformation of the biopolymers. It is revealed that the temperature range of volume phase transition is (1) primarily a function of the doping ratio of thermoresponsive polymer in the hybrid matrix, and is (2) sensitive to the Flory-Huggins interaction parameter between the biopolymer and water. This model can be a simple and robust tool for precisely designing intelligent tissue-like hydrogel materials and devices.

\section{Acknowledgments}

This research was supported by the National Research Council of Science \& Technology (NST) grant by the South Korean government (MSIP) (No. CAP-17-01-KIST Europe) and Project 11911. We thank Dr. Chanjoong Kim (Liquid Crystal Institute, Kent, OH, USA) for his advice and helpful discussion.

\section{References}


1. De Gennes P-G. Scaling Concepts in Polymer Physics . Ithaca, NY, USA: Cornell University Press; 1979.

2. Peppas NA, Hilt JZ, Khademhosseini A, Langer R. Hydrogels in biology and medicine: from molecular principles to bionanotechnology. Adv Mater. 2006; 18:1345-1360.

3. Slaughter BV, Khurshid SS, Fisher OZ, Khademhosseini A, Peppas NA. Hydrogels in regenerative medicine. Adv Mater. 2009; 21:3307-3329.

4. Schild HG. Poly(N-isopropylacrylamide): experiment, theory and application. Prog Polym Sci. 1992; 17:163-249.

5. Huggins ML. Solutions of long chain compounds. J Chem Phys.1941; 9:440.

6. Flory PJ. Thermodynamics of high polymer solutions. J Chem Phys. 1942; 10:51-61.

7. Flory PJ, Rhener J. Statistical mechanics of cross-linked polymer networks. J Chem Phys. 1943; 11:512520.

8. Flory PJ. Principles of Polymer Chemistry . Ithaca, NY, USA: Cornell University Press; 1953.

9. Shibayama M, Tanaka T. Volume phase transition and related phenomena of polymer gels. Adv Polym Sci. 1993; 109:1-62.

10. Annabi N, Tamayol A, Uquillas JA, Akbari M, Bertassoni LE, Cha C, Camci-Unal G, Dokmeci MR, Peppas NA, Khademhosseini A. 25th anniversary article: rational design and applications of hydrogels in regenerative medicine. Adv Mater. 2014; 26:85-124.

11. Russel TH, Edwards BJ, Khomami B. Characterization of the Flory-Huggins interaction parameter of polymer thermodynamics. Europhys Lett. 2014; 108:66003.

12. Lopez CG, Richtering W. Does Flory-Rhener theory quantitatively describe the swelling of thermoresponsive microgels? Soft Matter 2017; 13:8271-8280.

13. Lietor-Santos J-J, Sierra-Martin B, Vavrin R, Hu Z, Gasser U, Fernandez-Nieves A. Deswelling microgel particles using hydrostatic pressure. Macromolecules 2009; 42:6225-6230.

14. Dewhirst MW, Viglianti BL, Lora-Michiels M, Hanson M, Hoopes PJ. Basic principles of thermal dosimetry and thermal thresholds for tissue damage from hyperthermia. Int $J$ Hyperthermia 2003; 19:267-294.

15. Wallace DG. The role of extrahelical peptides in stabilization of collagen fibrils. Biopolymers 1990; 30:889-897.

16. Bohidar HB. Hydrodynamic properties of gelatin in dilute solutions.Int J Biol Macromol. 1998; 23:1-6.

17. Bohidar HB, Jena SS. Kinetics of sol-gel transition in thermoreversible gelation of gelatin. $J$ Chem Phys. 1993; 98:8970-8977.

18. Bozec L, Odlyha M. Thermal denaturation studies of collagen by microthermal analysis and atomic force microscopy. Biophys J.2011; 101:228-236.

19. Sung B, Kim C, Kim M-H. Biodegradable colloidal microgels with tunable thermosensitive volume phase transitions for controllable drug delivery. J Colloid Interface Sci. 2015; 450:26-33.

20. Lynn AK, Yannas IV, Bonfield W. Antigenicity and immunogenicity of collagen. J Biomed Mater Res Part B: Appl Biomater. 2004; 71B:343-354.

Table 1: Constant parameters and a semi-fixed variable used in the model.

\begin{tabular}{llll}
\hline Symbol & Definition & Value & Remark \\
\hline$v_{w}$ & $\begin{array}{l}\text { Volume of a lattice }(= \\
\text { volume of a } \mathrm{H}_{2} \mathrm{O} \\
\text { molecule in liquid } \\
\text { phase) }\end{array}$ & Constant \\
& $\begin{array}{l}\text { Initial volume of a gel } \\
\text { in a fully swollen state } \\
\text { Volume fraction of } \\
V_{i}\end{array}$ & $1.0 \times 10^{-29} \mathrm{~m}^{3}$ & \\
$\varphi_{\text {NIIIA }}$ & pNIPAM $\left(=\mathrm{m}_{\text {NIPA }} / V_{i}\right)$ & Constant & Constant
\end{tabular}




\begin{tabular}{|c|c|c|c|}
\hline Symbol & Definition & Value & Remark \\
\hline $\bar{A}$ & $\begin{array}{l}\text { Elasticity-associated } \\
\text { coefficient } \\
\text { (dimensionless) }\end{array}$ & $-6.4^{[13]}$ & Constant \\
\hline$\Theta$ & $\begin{array}{l}\text { Flory's } \Theta \text { temperature } \\
\text { of pNIPAM }\end{array}$ & $315 \mathrm{~K}^{[13]}$ & Constant \\
\hline$\chi_{\beta \imath o}$ & $\begin{array}{l}\text { Flory-Huggins interaction } \\
\text { parameter between } \\
\text { tissue-derived biopolymer } \\
\text { and water } \\
\text { (dimensionless) }\end{array}$ & $\begin{array}{l}0.161 \text { for intact } \\
\text { (composite) collagen type } \\
\mathrm{I}^{[15]} 0.107 \text { for } \\
\text { pronase-digested collagen } \\
\text { type } \mathrm{I}^{[15]} 0.48 \text { for a } \\
\text { gelatin coil }{ }^{[17]} 0.49 \text { for } \\
\text { gelatin in coil }+ \text { helical } \\
\text { states }[17,18]\end{array}$ & $\begin{array}{l}\text { Semi-fixed (4 different } \\
\text { values) }\end{array}$ \\
\hline
\end{tabular}

Table 2: Model variables for numerical plotting.

\begin{tabular}{ll}
\hline Symbol & Definition \\
\hline$a$ & Gel elasticity-associated coefficient (dimensionless) \\
$T$ & Temperature (K) \\
$N_{x}$ & Degree of polymerization between crosslinks in a network (dimensionless). $0<N_{x}$. \\
$V / V_{i}$ & Gel volume ratio with reference to the initially (fully) swollen state (dimensionless). For deswelling (shrinking), 0 \\
$r_{\text {polym }}$ & Doping ratio of thermoresponsive pNIPAM to tissue-derived biopolymer in a gel (dimensionless). $0<r_{\text {polym }}=V$ \\
\hline
\end{tabular}

\section{Figure Legends}

Figure 1: Schematic drawing of a hybrid polymer network in a swollen state.Figure 2: Dependence of the type I collagen gel volume change on the elasticity-associated coefficient $\alpha$, as a function of normalized temperature, $T / \Theta$. Other physicochemical parameters are fixed at $r_{p o l y m}$ $=1, N_{x}=1$, and $\chi_{\beta \imath o}=0.161$.Figure 3: Effect of the doping ratio $\left(r_{\text {polym }}\right)$ of thermoresponsive pNIPAM $(\Theta=315 \mathrm{~K})$ on the volume phase transition temperature of a type I collagen gel $\left(\chi_{\beta 1 o}=0.161\right)$. Four values of $r_{\text {polym }}$ are presented as an example: $r_{\text {polym }}=0.50,0.40,0.35$, and 0.30 from the left, with fixed $a=1.0 \times 10^{7}$. The secondary dependence on the crosslinking degree is displayed as decreasing saturation for each $r_{\text {polym }}$ value, from $N_{x}=1$ to 5 . Room temperature: $T_{\text {room }}$; human body temperature: $T_{\text {body }}$.Figure 4: Effect of type I collagen (and gelatin) molecular structure (represented by $\chi_{\beta}$ ) on the transition temperature of a hybrid gel. With the fixed parameters of $a=1.0 \times 10^{7}, r_{\text {polym }}=0.35$, and $N_{x}=1$, the volume phase transition curves are plotted for four different biopolymer structures in the hybrid gel, that is, two rod-like conformations of collagen type ${ }^{20}\left(\chi_{\beta \imath o}=0.161\right.$ for "intact composite" helical rod with extrahelical peptides, blue solid line; and $\chi_{\beta 10}=0.107$ for "pronase-digested" pure helical rod, blue dashed line) and two conformational states of gelatin chains $\left(\chi_{\beta 10}=0.48\right.$ for coil state, cyan solid line; and $\chi_{\beta 10}=0.49$ for coil + helical states, cyan dashed line). Thermal denaturation temperature from type I collagen to gelatin is denoted as $T_{\text {col-gel }}$.Figure 5: Optimized sets of dimensionless parameters for the hydrogel matrices of type I collagen and gelatin where thermoresponsive pNIPAM chains are doped. For collagen (blue solid line), $\left\{V_{i} / V_{N I P A}, a, N_{x}, r_{\text {polym }}, \chi_{\beta 10}\right\}$ $=\left\{20,1.0 \times 10^{7}, 1,0.35,0.161\right\}$. For gelatin (cyan dotted line), $\left\{V_{i} / V_{N I P A}, a, N_{x}, r_{\text {polym }}, \chi_{\beta 10}\right\}=$ $\left\{20,1.0 \times 10^{7}, 1,0.48,0.49\right\}$. The parameter values are chosen to enable the gel matrices remain fully swelled at $T_{b o d y}$ and to enable complete shrinking within hyperthermal ranges where the tissues are not damaged. 

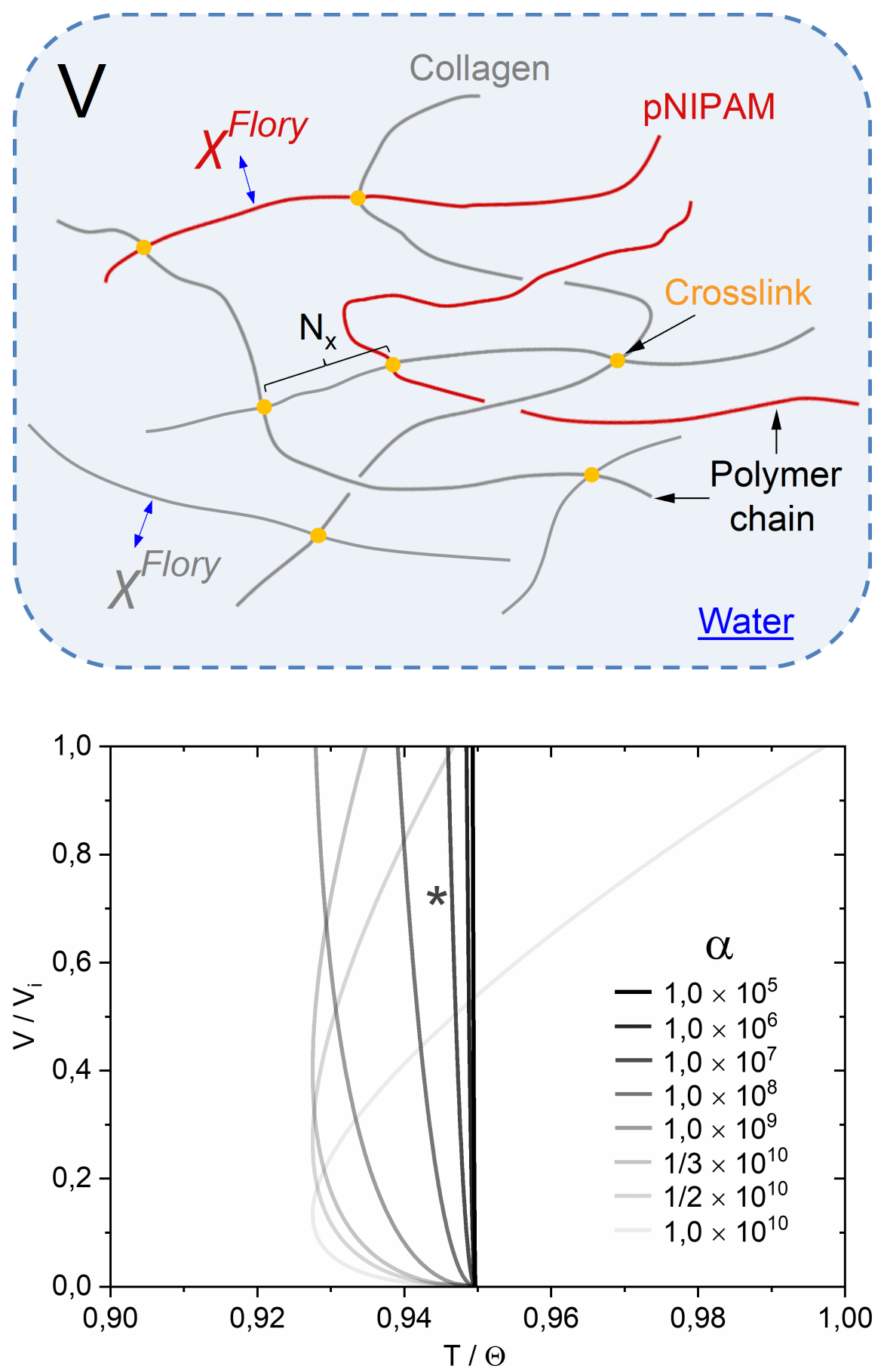

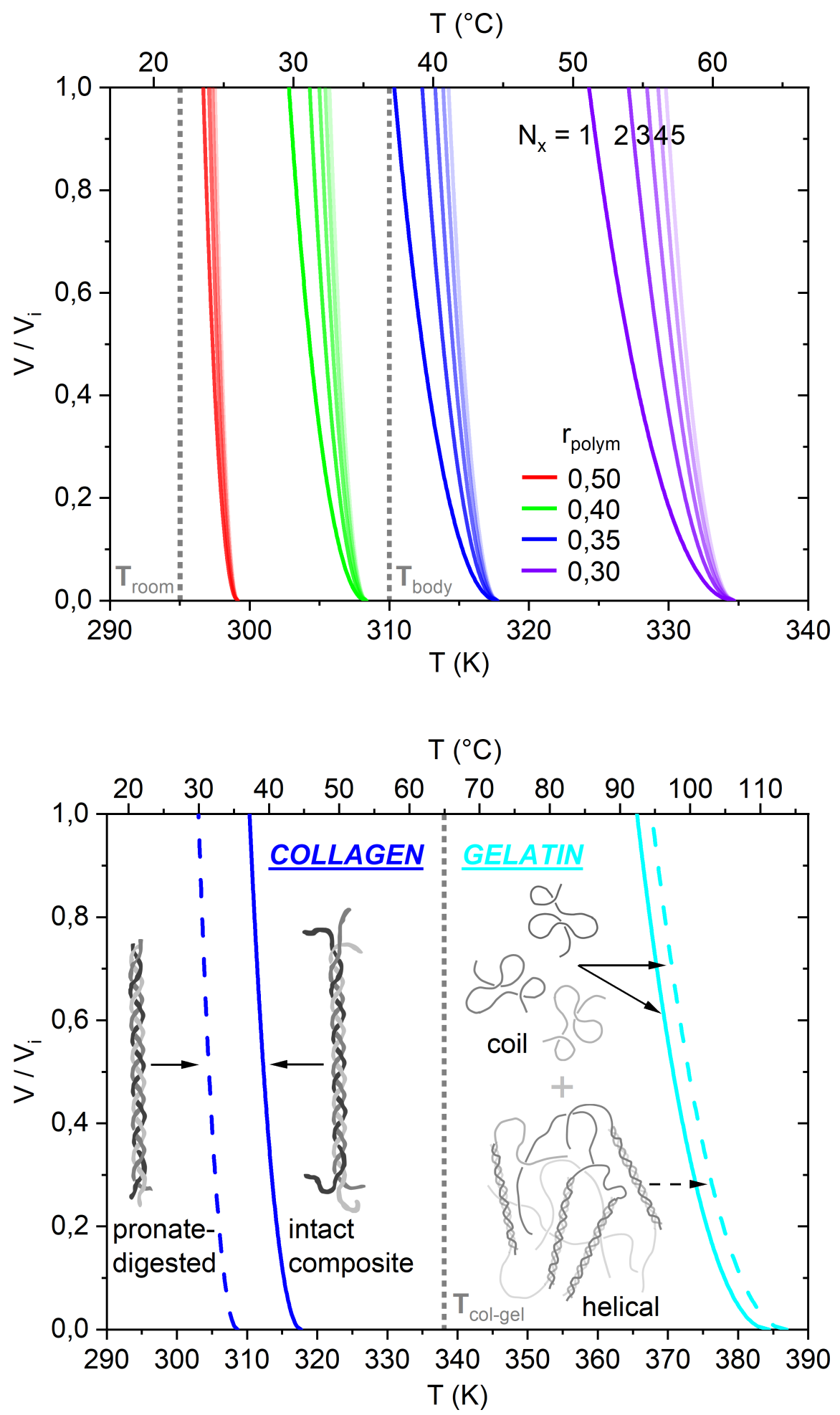


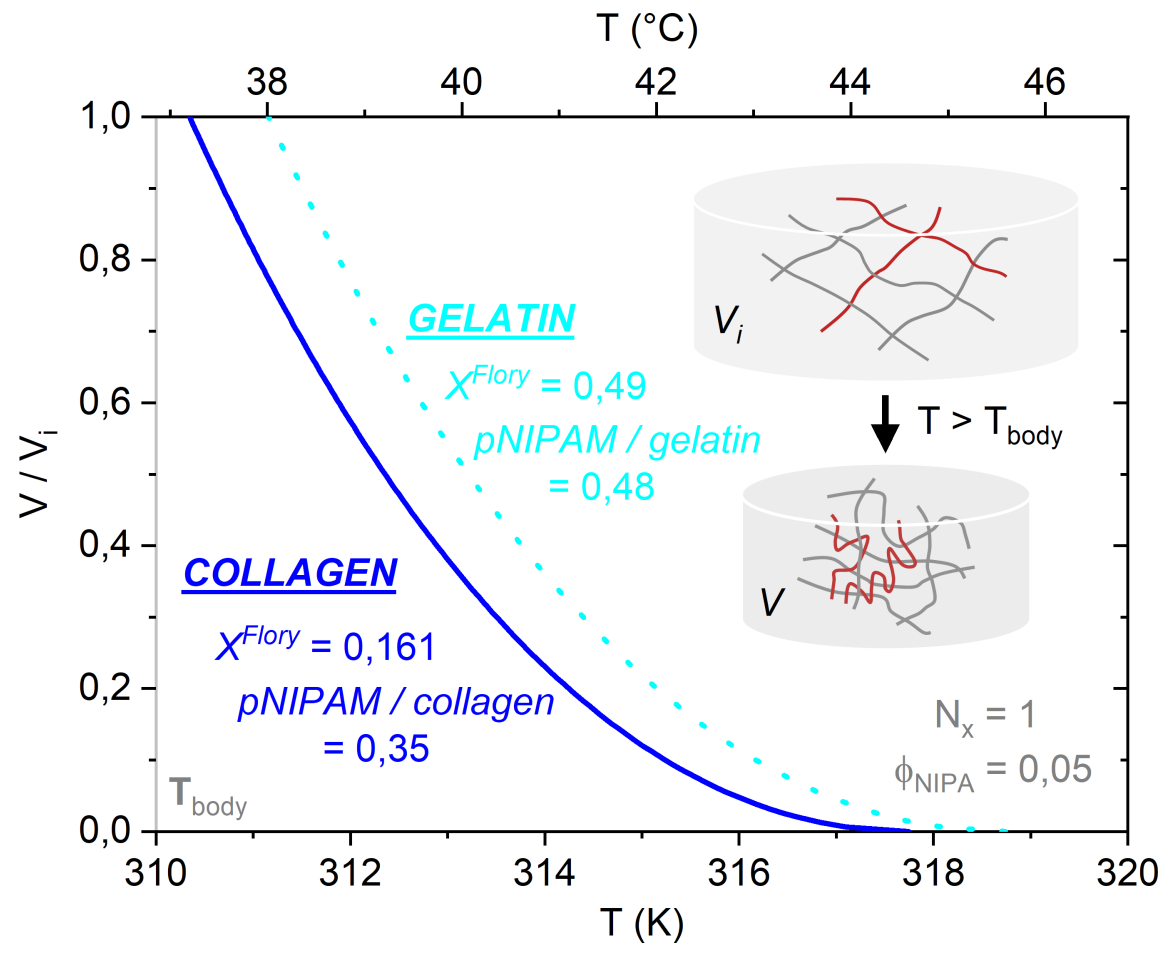

